\title{
LES RÉSULTATS DE LA CONFÉRENCE INTERGOUVERNEMENTALE 2003-2004
}

Franklin Dehousse, Wouter Coussens, Jordi Garcia et Pierre Van Den Brule

CRISP | « Courrier hebdomadaire du CRISP »

2004/22 $n^{\circ} 1847$ | pages 1 à 39

ISSN 0008-9664

Article disponible en ligne à l'adresse :

https://www.cairn.info/revue-courrier-hebdomadaire-du-crisp-2004-22-page-1.htm

Distribution électronique Cairn.info pour CRISP.

(C) CRISP. Tous droits réservés pour tous pays.

La reproduction ou représentation de cet article, notamment par photocopie, n'est autorisée que dans les limites des conditions générales d'utilisation du site ou, le cas échéant, des conditions générales de la licence souscrite par votre établissement. Toute autre reproduction ou représentation, en tout ou partie, sous quelque forme et de quelque manière que ce soit, est interdite sauf accord préalable et écrit de l'éditeur, en dehors des cas prévus par la législation en vigueur en France. Il est précisé que son stockage dans une base de données est également interdit. 
Courrier hebdomadaire

$\mathrm{n}^{\circ} 1847 \cdot 2004$

\section{Les résultats de la Conférence intergouvernementale 2003-2004}

Une évaluation à la lumière du projet établi par la Convention

Franklin Dehousse

Wouter Coussens

Jordi Garcia

Pierre Van Den Brule

Centre de recherche et d'information socio-politiques 


\title{
CENTRE DE RECHERCHE ET D'INFORMATION SOCIO-POLITIQUES - CRISP
}

\author{
Président : Xavier Mabille \\ Directeur général : Vincent de Coorebyter \\ Secrétaire général : Pierre Blaise \\ Rédacteur en chef : Étienne Arcq \\ Équipe de recherche : \\ Étienne Arcq, Pierre Blaise, Vincent de Coorebyter, \\ Caroline Sägesser, Anne Vincent, Marcus Wunderle
}

\section{Conseil d'administration :}

Jacques Brassinne de La Buissière (vice-président), Camille Deguelle, Franklin Dehousse (en congé), Francis Delpérée, Hugues Dumont, Jacques Emond, Jules Gérard-Libois (président fondateur), José Gotovitch, Nadine Gouzée, Serge Govaert, Maurice-Pierre Herremans (président honoraire), Laura Iker, Malou Julin, Xavier Mabille (président), Roland Michel (administrateur gérant), Pierre Reman, Guy Spitaels, Robert Tollet (vice-président), Els Witte, Paul Wynants

\section{COURRIER HEBDOMADAIRE}

Le numéro simple : 6,90€- Le numéro double : 12,40€

Abonnement : 235,00€

(TVA comprise)

\author{
Place Quetelet, 1A - 1210 Bruxelles - Tél.: 02/211 0180 \\ Banque : 310-0270551-07 - CCP : 000-0065824-58 \\ Site web : www.crisp.be
}

Le Courrier hebdomadaire bénéficie des remarques et suggestions de l'équipe de recherche du CRISP. Les projets de textes sont également soumis à la lecture de spécialistes extérieurs à l'équipe de recherche, et qui sont choisis en fonction des sujets traités.

Le Courrier hebdomadaire est membre de l'Association des revues scientifiques et culturelles (ARSC).

Éditeur responsable : V. de Coorebyter, Place Quetelet, 1A - 1210 Bruxelles

Tous droits de traduction, d'adaptation ou de reproduction par tous procédés, y compris la photographie et le microfilm, réservés pour tous pays

ISSN 00089664 


\section{Table des matières}

$\begin{array}{lr}\text { INTRODUCTION } & \mathbf{5}\end{array}$

1. UN NOUVEAU PROCESSUS

1.1. Le projet établi par la Convention : un bilan mitigé $\mathbf{6}$

$\begin{array}{ll}\text { 1.2. La Conférence intergouvernementale } & 7\end{array}$

2. LES INSTITUTIONS 9

2.1. Le Conseil 9

2.1.1. La définition de la majorité qualifiée 9

2.1.2. La portée du vote à la majorité qualifiée 11

2.1.3. Les formations du Conseil 12

2.1.4. La présidence du Conseil 12

2.2. La Commission 13

2.2.1. La composition de la Commission 13

2.2.2. La nomination de la Commission $\quad 15$

2.3. Le Parlement européen $\quad 15$

2.4. Le ministre des Affaires étrangères 16

2.5. La coopération renforcée 17

3. LES POLITIQUES

$\begin{array}{ll}\text { 3.1. Les droits fondamentaux } & 18\end{array}$

3.1.1. Les explications relative à la Charte 18

3.1.2. L'adhésion à la CEDH 19

3.2. L'Union économique et monétaire 19

3.2.1. Le pilier monétaire 19

3.2.2. La reconnaissance de la spécificité de la zone euro dans
le processus décisionnel du Conseil

3.2.3. Les prérogatives de la Commission dans la procédure de déficit excessif 20

3.3. La sécurité intérieure $\quad 21$

3.3.1. Eurojust 22

3.3.2. Le procureur européen $\quad 22$

3.3.3. Règles communes minimales dans le secteur du droit pénal 22

3.3.4. Le protocole sur la position du Royaume-Uni et de l'Irlande 23

$\begin{array}{ll}\text { 3.4. Les relations extérieures } & 24\end{array}$

3.4.1. La coopération structurée permanente 24

3.4.2. La défense mutuelle 26

3.4.3. La procédure de décision dans la politique commerciale commune 27

3.4.4. Le service européen pour l'action extérieure 28

3.4.5. La compétence de la Cour de justice dans le domaine PESC 28

$\begin{array}{ll}\text { 3.5. La politique sociale } & 28\end{array}$

3.5.1. La clause sociale horizontale 28

3.5.2. Le recul en matière de protection sociale des travailleurs migrants 29

3.5.3. Les services d'intérêt économique général 29

$\begin{array}{ll}\text { 3.6. La fiscalité } & \mathbf{3 0}\end{array}$ 
4.1. Les ressources propres $\quad 31$

4.2. Le cadre financier pluriannuel 31

4.3. Le budget annuel $\quad 32$

CONCLUSION $\quad 34$

Une régression légère mais certaine $\quad 34$

Un système institutionnel légèrement plus complexe et obscur 34

Des politiques légèrement moins efficaces $\quad 35$

Une procédure de révision dont la réforme s'impose 36 


\section{INTRODUCTION}

Le 18 juin 2004, les chefs d’État et de gouvernement des États membres, réunis à Bruxelles, ont conclu les négociations sur le Traité établissant une Constitution pour l'Europe. Notre étude portera sur une évaluation des résultats de la Conférence intergouvernementale à la lumière du projet qui avait été établi par la Convention européenne ${ }^{1}$. La première partie esquissera le processus ayant mené à l'accord sur le Traité constitutionnel. Les parties deux, trois et quatre compareront l'accord final avec le projet de la Convention en ce qui concerne la structure institutionnelle, les différentes politiques et les dispositions budgétaires. La conclusion est consacrée à une évaluation synthétique du Traité, notamment au regard de la complexité du système institutionnel et de l'efficacité des politiques de l'Union. On s'interrogera enfin sur l'avenir de la procédure de révision.

F. Dehousse est juge au Tribunal de première instance des Communautés européennes, assesseur des recherches européennes à l'IRRI et professeur extraordinaire à l'Université de Liège. W. Coussens, J. Garcia et P. Van Den Brule sont chercheurs à l'IRRI. Le présent commentaire n'engage que les auteurs, et non les institutions auxquelles ils appartiennent.

1 Cf. aussi D. PHINNEMORE, "The Treaty establishing a Constitution for Europe: an overview », Chatham House Briefing Note, June 2004; G. GREVI, "Light and Shade of a quasi-Constitution. An assessment », EPC Issue Paper, nº 14, 2004. 


\section{UN NOUVEAU PROCESSUS}

\subsection{LE PROJET ÉTABLI PAR LA CONVENTION : UN BILAN MITIGÉ}

Au Conseil européen de Laeken de 2001, à l'instigation de la présidence belge, les leaders européens adoptèrent la déclaration dite de Laeken. Les chefs d'État et de gouvernement y reconnaissaient que le Traité de Nice ne pouvait garantir un fonctionnement efficace et démocratique satisfaisant de l'Union au sein d'une Europe élargie ${ }^{2}$. La décision fut par conséquent prise de réviser ce traité afin de rendre l'Union plus transparente, démocratique et efficace. Dans ce but, un nouvel organe, «la Convention européenne», fut créé. Il reçut la tâche de préparer un projet de texte à la Conférence intergouvernementale ${ }^{3}$. Ce texte a été présenté en juillet $2003^{4}$.

Après examen, le bilan de la Convention apparaissait plutôt mitigé, au regard des objectifs de transparence, de démocratie et d'efficacité exaltés par le Conseil européen ${ }^{5}$.

Certes, le projet de traité renforce la transparence de l'Union par une simplification de la structure du traité, une réforme des instruments juridiques et des procédures, une catégorisation des compétences et la création d'un Conseil législatif. Toutefois, cette simplification aurait pu être plus poussée. Le cadre institutionnel et politique de l'Union demeure, en effet, dans de nombreux secteurs, trop complexe. Certaines complexités nouvelles ont même été ajoutées.

De la même manière, le projet de traité renforce la démocratie au niveau européen. Il faut reconnaitre le renforcement des pouvoirs législatifs et budgétaires du Parlement européen, la participation plus importante des parlements nationaux, l'instauration d'un droit d'initiative populaire et l'incorporation de la Charte des droits fondamentaux dans le traité. Toutefois, cette démocratisation n'apparait pas complète. Le rôle de surveillance du Parlement européen reste dans plusieurs secteurs importants marginal. Le veto des

2 F. Dehousse, «La CIG 2000 : vers une réforme incomplète des institutions européennes ? », Courrier hebdomadaire, CRISP, $\mathrm{n}^{\circ} 1674,2000$.

3 F. DEHOUSSE, «Le débat sur l'avenir de l'Europe et la déclaration de Laeken », JT, 2002, pp. 297301.

4 JOCE 2003, C 169.

5 Il convient de reporter à des analyses plus complètes : cf. F. DEHOUSSE et W. COUSSENS (réd.), "The Convention's Constitutional Treaty: old wine in a new bottle ?", Studia Diplomatica, n 1-2, 2003 ; F. DeHOusse et W. Coussens, «Le Traité Constitutionnel de la Convention pour l'Europe: un nouveau pas pour l'intégration européenne? ", Courrier hebdomadaire, CRISP, nº 1808-1809, 2003. Sur les travaux de la Convention, cf. notamment A. LAMASSOURE, Histoire secrète de la Convention européenne, Paris, Albin Michel, 2004; A. DAUVERGNE, L'Europe en otage? Histoire secrète de la Convention, Paris, Saint-Simon, 2004 ; P. NORMAN, The accidental Constitution - The story of the European Convention, Brussels, Eurocommont, 2003. Sur le projet de la Convention, cf. K. LENAERTS, J.M. BINON et P. VAN NUFFEL, «L'Union européenne en quête d'une Constitution : bilan des travaux de la Convention sur l'avenir de l'Europe», JTDE, 2003, pp. 289-298 ; J. KOKOTT and A. RUTH, «The European Convention and its draft treaty establishing a Constitution for Europe: appropriate answers to the Laeken questions?", CML Rev, 2003, pp. 1315-1345; M. DOUGAN, "The Convention's draft constitutional treaty: bringing Europe closer to its lawyers ", ELJ, 2003, pp. 763793 ; C. PHILIP et P. SOLDATOS dirs., La Convention sur l'avenir de l'Europe, Bruxelles : Bruylant, 2004. 
États membres, qui peut entraver des majorités énormes, demeure dans une série de matières stratégiques. Enfin, la procédure de révision des traités demeure fortement intergouvernementale.

Enfin, le projet de traité de la Convention confère à certains égards plus d'efficacité à l'Union, mais ce progrès demeure très modeste. Il faut reconnaitre l'extension du vote à la majorité qualifiée, le renforcement substantiel opéré en matière de sécurité intérieure et la possibilité ouverte à un groupe limité d'États membres de "prendre l'initiative». Toutefois, ici, la Convention aurait pu aller beaucoup plus loin. L'unanimité reste en effet, par exemple, la règle en matière de politique étrangère, de politique sociale, de taxation et de révision future de la Constitution. L'efficacité de l'Union est par ailleurs encore sapée par certaines propositions en matière institutionnelle, la plus importante étant la création d'un président du Conseil européen dont les fonctions risquent de provoquer une certaine concurrence entre celui-ci, le ministre des Affaires étrangères et le président de la Commission européenne.

Sur le plan des institutions européennes, la Convention a entrepris la réforme la plus importante depuis 1957. Celle-ci change l'équilibre actuel en favorisant clairement l'aspect intergouvernemental de l'Union. Le Conseil européen, reconnu comme réelle institution de l'Union, voit ses prérogatives étendues. Il sera de plus dirigé par un président permanent. Associé au nouveau poste de ministre des Affaires étrangères, cette nouvelle fonction risque de renforcer l'aspect intergouvernemental de la politique extérieure européenne tout comme d'ailleurs l'introduction d'une Commission à deux niveaux.

Sur le plan politique, enfin, la Convention a apporté deux surprises, l'une positive et l'autre négative. La surprise positive réside dans l'absence de conflit structurel entre les anciens et les nouveaux États membres. La Convention constituait la première négociation, certes de nature spécifique, menée avec des représentants de 28 États (il convient de ne pas oublier qu'elle comprenait des représentants de tous les États candidats, en ce compris de la Turquie). Elle a certainement constitué un succès à cet égard. En revanche, la surprise négative réside dans la résurgence, et même l'intensification de l'affrontement entre les grands et les petits États membres. De manière révélatrice, les deux thèmes de négociation les plus difficiles ont été la réforme des modalités du vote à la majorité qualifiée et la réforme de la composition de la Commission. Cela avait déjà été le cas lors de la CIG de 2000, achevée à Nice. Cela explique d'ailleurs la décision de reporter les réformes dans ces domaines, ainsi que pour la composition du Parlement européen. La Convention a en outre ajouté par ses propositions relatives à la présidence du Conseil et du Conseil européen un nouveau champ de bataille à cette « lutte» entre les petits et les grands États membres.

\subsection{LA CONFÉRENCE INTERGOUVERNEMENTALE}

Il faut tout d'abord rappeler que le texte préparé par la Convention constituait seulement un projet. Ce projet présentait toutefois une grande importance puisqu'il formait la base 
à partir de laquelle la Conférence intergouvernementale (CIG) devait atteindre un accord final sur le Traité constitutionnel ${ }^{6}$.

Cette Conférence a commencé en octobre $2003^{7}$. Initialement, tout s'y déroula comme prévu laissant augurer une conclusion de ses débats pour la fin de l'année. Le Conseil européen de décembre échoua toutefois à obtenir un accord unanime des États membres sur un texte. Trois raisons ont souvent été invoquées pour expliquer cet échec : une présidence italienne mal gérée, l'intransigeance de l'Espagne et de la Pologne sur la question de la majorité qualifiée et l'attitude ambivalente de la France et du Royaume-Uni. Ce dernier était en effet hanté par la perspective d'un référendum à propos de la Constitution et insistait dès lors avec virulence sur ce qu'il appelle ses «lignes rouges» (les points sur lesquels il ne peut accepter le moindre compromis). Le président Chirac, quant à lui aussi soumis à la menace d'un référendum, paraissait heureux de pouvoir démontrer l'extrême difficulté d'un processus décisionnel à 25 ainsi que la nécessité de « groupes de pionniers ».

Il est extrêmement intéressant de noter que la négociation paraissait définitivement dans l'impasse, jusqu'aux attentats de Al-Qaeda en Espagne en mars 2004. Cette manifestation d'une menace terroriste nouvelle dans son ampleur provoqua une sorte de retour à la raison. Le changement de gouvernement en Espagne facilita encore davantage la reprise des travaux. Conduits par une présidence irlandaise particulièrement agile, ceux-ci débouchèrent finalement le 18 juin 2004 sur l'adoption d'un traité établissant une Constitution pour l'Europe par les chefs d'État et de gouvernement ${ }^{8}$. Ce traité doit maintenant être ratifié par l'ensemble des 25 États membres pour pouvoir entrer en vigueur.

Le projet de traité adopté par la CIG conserve la structure générale en quatre parties du projet présenté par la Convention. Toutefois, il a été complété par des protocoles et déclarations couvrant plusieurs centaines de pages. L'ensemble apparait par conséquent singulièrement compliqué au lecteur moyen. En moyenne, la plupart des dispositions du projet de la Convention ont subi peu de changements. Toutefois, le nombre et la portée stratégique des modifications apportées aboutissent à modifier de manière substantielle la portée du traité.

6 Cf. les conclusions du Conseil européen de Thessalonique (20 juin 2003).

7 Un groupe d'experts a été mis en place pour «nettoyer» le projet de la Convention. Le groupe a produit quelques clarifications et corrections de texte intéressantes. Cf. CIG 4/03 et CIG 50/03.

8 Cf. les documents CIG 50/03, CIG 81/04 et CIG 85/04. Une version consolidée du Traité établissant une Constitution pour l'Europe peut être trouvée dans le document CIG 87/04. Les protocoles annexés au Traité constitutionnel se trouvent dans le document CIG 87/04 ADD 1, et les déclarations à annexer à l'acte final de la Conférence intergouvernementale dans le document CIG 87/04 ADD2. 


\section{LES INSTITUTIONS}

\subsection{LE CONSEIL}

\subsubsection{La définition de la majorité qualifiée}

La Convention proposait de remplacer le système complexe, opaque et inefficace de la majorité qualifiée adopté à Nice ${ }^{9}$ par un système de double majorité ${ }^{10}$. Ce changement ne devait toutefois entrer en vigueur qu'en 2009. Au lieu de la triple majorité de Nice, dans ce nouveau système, une majorité qualifiée était acquise dès lors que la moitié des États membres représentant $60 \%$ de la population de l'Union soutenait la mesure en cause. Dans le cas particulier où le Conseil n'aurait pas agi sur la base d'une proposition de la Commission ou du ministre des Affaires étrangères, la majorité qualifiée exigée consistait alors en deux tiers des États membres, représentant au moins trois cinquièmes de la population de l'Union. Comparé au Traité de Nice, ce système présentait le double avantage d'être plus transparent et d'assurer un renforcement de la capacité d'action de l'Union. Toutefois, cette technique renforçait également considérablement la puissance de vote des États membres les plus peuplés.

La CIG a abouti à un système nettement plus compliqué que celui de la Convention ${ }^{11}$. Une majorité qualifiée requiert au moins $55 \%$ des membres du Conseil, comprenant au moins quinze États membres et représentant au moins $65 \%$ de la population de l'Union. L'exigence des quinze États membres sera toutefois très probablement superflue dès 2009, puisque dans une Union à 27, $55 \%$ des États membres comprendront nécessairement 15 États membres. De plus, dans ce système, une minorité de blocage doit inclure au moins quatre membres du Conseil, faute de quoi la majorité qualifiée est réputée acquise. Cette dernière condition a été insérée pour limiter le poids des pays les plus peuplés. Toutefois, elle pourrait mener à une conséquence étrange, à savoir qu'elle pourrait permettre à une décision d'être adoptée à une majorité ne rassemblant pas $65 \%$ de la population ${ }^{12}$.

Dans l'hypothèse où le Conseil n'agit pas sur une proposition de la Commission ou du ministre des Affaires étrangères, le système mis en place par la CIG prévoit que la

9 Dans le cadre du Traité de Nice (article 205 CE), la majorité qualifiée implique, dès le per janvier 2005, que la décision en cause recueille au moins 232 voix sur 321 exprimant soit le vote favorable de la majorité des membres, lorsqu'en vertu du traité, elle doit être prise sur proposition de la Commission, soit le vote favorable d'au moins deux tiers des membres, dans les autres cas. Pour ce faire, les voix des membres du Conseil sont affectées d'une pondération complexe. Un membre du Conseil peut en outre demander que, lors de la prise d'une décision par le Conseil à la majorité qualifiée, il soit vérifié que les États membres constituant cette majorité qualifiée représentent au moins $62 \%$ de la population totale de l'Union. Si cette condition n'est pas remplie, la décision n'est pas adoptée.

10 Cf. CEPR, « Nice try: should the Treaty of Nice be ratified ? ", Monitoring European Integration, $\mathrm{n}^{\circ} 11$ (2001).

11 Article I-25.

12 J. EMMANOUILIDIS, " Historically unique, unfinished in detail - an evaluation of the Constitution ", Centre for Applied Policy Research Reform Spotlight, 2004/03. 
majorité qualifiée correspond à $72 \%$ des membres du Conseil, représentant des États membres comprenant au moins $65 \%$ de la population de l'Union. Cette majorité « renforcée » est applicable à des mesures aussi importantes que les décisions du Conseil en matière de Politique étrangère et de sécurité commune (PESC) lorsque celui-ci y agit sur son initiative propre, les décisions dans le cadre de l'Union économique et monétaire (UEM) fondées sur une recommandation de la Commission ou de la Banque centrale européenne ou les nominations.

En entrant des nombres absolus dans le système, la CIG l'a rendu moins flexible. En effet, un des avantages d'un système de double majorité tel qu'envisagé par la Convention est qu'il s'adapte automatiquement aux évolutions. Il n'y a dès lors plus besoin de le modifier en cas d'élargissement ou de situations dans lesquelles tous les membres n'ont pas le droit de vote (comme en matière d'UEM, de coopération renforcée ou de coopération structurée, par exemple). En insérant des nombres dans le système, la CIG a fait disparaitre ces avantages. Dans les domaines dans lesquels seuls quelques États membres ont le droit de vote, par exemple, les dispositions de la Constitution devront par conséquent définir de manière spécifique la majorité qualifiée

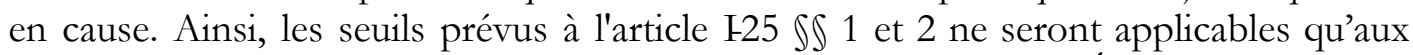
membres du Conseil qui ont le droit de vote et à la population des États membres qu'ils représentent et une minorité de blocage devra dans ce cas inclure au moins le nombre minimal de membres du Conseil représentant plus de $35 \%$ de la population des États membres participants, plus un membre, faute de quoi la majorité qualifiée sera réputée acquise. L'exigence qu'une majorité qualifiée comprenne au moins quinze États membres sera dans ces cas supprimée.

De plus, le système a encore été rendu plus complexe par l'adjonction d'un compromis semblable à celui de Ioannina ${ }^{13}$. Ainsi, si des membres du Conseil, représentant au moins trois quarts de la population, ou au moins trois quarts du nombre d'États membres, requis pour constituer une minorité de blocage, indiquent leur opposition au fait que le Conseil adopte un acte à la majorité qualifiée, le Conseil est tenu de débattre $\mathrm{du}$ problème et de faire tout ce qui est en son pouvoir pour trouver une solution satisfaisante aux préoccupations soulevés par ces États membres. Ce compromis est censé entrer en vigueur le $1^{\text {er }}$ novembre 2009 et rester en application au moins jusqu'en 2014. Après cette date, le Conseil aura en effet la possibilité d'adopter une décision européenne l'abrogeant ${ }^{14}$.

13 Le compromis de Ioannina tire son nom d'une réunion informelle des ministres des Affaires étrangères à Ioannina, en Grèce, le 29 mars 1994. De cette réunion a résulté, entre autres choses, une décision concernant la question du vote à la majorité qualifiée au Conseil après l'élargissement des pays AELE. Le compromis atteint prévoit que si des membres du Conseil représentant un total de 23 (ancien seuil de la minorité de blocage) à 26 voix (nouveau seuil de la minorité de blocage) indiquent leur intention de s'opposer à la prise d'une décision par le Conseil à la majorité qualifiée (c'est-à-dire, à l'époque, par une majorité de 64 voix et donc une minorité de blocage de 27 voix), le Conseil fait tout ce qui est en son pouvoir pour aboutir, dans un délai raisonnable et sans porter préjudice aux limites obligatoires de temps fixées par les traités et le droit dérivé, à une solution satisfaisante qui puisse être adoptée par 65 voix sur 87 au moins. Le Traité de Nice a mis fin à ce compromis.

14 CIG 87/04 ADD 2, Déclaration ad article I-25. 
Il ressort par conséquent de ces développements que le système adopté par la CIG est moins transparent et moins efficace que celui de la Convention. En augmentant les deux seuils (nombre d'États membres et volume de la population) et en ajoutant de nouvelles conditions, la capacité d'action de l'Union a été réduite de manière très significative, puisqu'il est désormais plus facile de former une minorité de blocage ${ }^{15}$. Cette règle de vote à la majorité qualifiée reste cependant légèrement plus efficace que le système de Nice, bien que l'efficacité en cas de majorité qualifiée « renforcée » ne soit pas beaucoup plus élevée que celle de Nice ${ }^{16}$. Toutefois, elle soulève des questions d'équité. Elle augmente en effet énormément la puissance de vote des grands États membres ${ }^{17}$. Cette augmentation vient par ailleurs s'ajouter au renforcement déjà important du pouvoir des grands États membres réalisé par le Traité de Nice. Les principaux perdants, comparés au système de Nice, sont dès lors d'une part les États membres «moyens » et d'autre part l'Espagne et la Pologne à qui toutefois on avait donné un pouvoir disproportionné à Nice. Le déplacement de la puissance de vote en faveur des États membres les plus peuplés peut enfin s'avérer être excessif dans les cas où (1) le Conseil n'agit pas sur la base d'une proposition de la Commission ou du ministre des Affaires étrangères, et/ou (2) tous les États membres n'ont pas le droit de vote, c'est-à-dire dans des secteurs aussi sensibles que l'UEM et la défense.

Notons enfin que le système proposé n'entrera en vigueur qu'en novembre $2009^{18}$. Le processus décisionnel au sein du Conseil risque ainsi d'être encore paralysé par le Traité de Nice pendant les cinq prochaines années ${ }^{19}$.

\subsubsection{La portée du vote à la majorité qualifiée}

La portée du vote à la majorité qualifiée a été revue par la CIG. Celle-ci en a d'une part réduit sa portée dans le domaine des taxes, mais aussi dans celui de certaines décisions budgétaires et indirectement dans les domaines de la sécurité sociale des travailleurs migrants et de la coopération judiciaire en matière pénale. D'autre part, elle l'a renforcée dans la coopération structurée en matière de défense (cf. infra).

La CIG a ensuite fait quelques modifications concernant les clauses «passerelle». Ces clauses sont des dispositions qui permettent au Conseil européen d'adopter une décision européenne autorisant le Conseil à statuer à la majorité qualifiée dans un domaine jusqu'alors soumis à l'unanimité, et/ou de statuer selon la procédure législative ordinaire

15 R. BALDWIN et M. WIDGREN, "Council voting in the Constitutional Treaty: Devil in the detail», CEPS Policy Brief, 2004.

16 À Nice, un très haut seuil de votes pondérés, le plus important des trois seuils, a été mis en place pour atteindre une majorité qualifiée. Ce système rend plus facile la formation d'une minorité de blocage et donc plus difficile la formation de majorités gagnantes. Le système de double majorité supprime le seuil de votes pondérés. Les seuils de population et de nombre d'États membres qu'il établit sont en outre relativement plus faciles à atteindre.

17 Notez cependant que la proposition de la Convention aurait encore plus favorisé les plus grands États membres. R. BALDWIN et M. WIDGREN, "Another failing in the making ", CEPS Commentary, 2004.

18 CIG 87/04 ADD 2, Protocole sur les dispositions transitoires relatives aux institutions et organes de l'Union.

19 Cf. l'article 2 du Protocole 34 sur les dispositions transitoires relatives aux institutions et organes de l'Union. 
dans un domaine jusqu'alors régi par une procédure législative spéciale. Le texte de la Convention contenait deux clauses passerelle générales. La première permettait au Conseil européen de décider unanimement de passer de l'unanimité à la majorité qualifiée dans une série de domaines de la Partie III de la Constitution. La deuxième permettait au Conseil européen d'autoriser le Conseil à statuer, dans la Partie III de la Constitution, selon la procédure législative ordinaire, à savoir la co-décision du Parlement et un vote à la majorité qualifiée au sein du Conseil, dans un domaine jusqu'alors régi par une procédure législative spéciale.

La CIG a rendu moins aisé le recours à ces clauses en prévoyant une procédure de décision plus contraignante. Toute initiative du Conseil européen sur la base de ces clauses devra en effet être notifiée aux parlements nationaux. Si un de ceux-ci fait connaitre son opposition dans les six mois de la date d'avis, la décision européenne ne pourra plus être adoptée ${ }^{20}$. L'utilisation de ces clauses est par ailleurs désormais exclue pour les décisions ayant des implications militaires ou en matière de défense.

\subsubsection{Les formations du Conseil}

Le projet d'un Conseil législatif, nouveauté de la Convention, fut l'une des premières victimes de la CIG. Cela paraît regrettable, dans la mesure où il aurait pu être un instrument important d'accroissement de la transparence et de la coordination des politiques européennes ${ }^{21}$. Les deux seules formations du Conseil encore mentionnées dans la Constitution sont dès lors le Conseil des Affaires générales et le Conseil des Affaires étrangères ${ }^{22}$. La séparation de ces deux formations demeure néanmoins une avancée à la lumière de la situation existante. Le Conseil européen adoptera par la suite à la majorité qualifiée la liste des autres formations.

\subsubsection{La présidence du Conseil}

La Convention avait abouti à un compromis assez obscur à propos de la question de la présidence du Conseil ${ }^{23}$. Selon le projet, le Conseil des Affaires étrangères devait être présidé par le ministre des Affaires étrangères. Pour les autres formations, un système de rotation égalitaire aurait été établi par le Conseil européen, prévoyant une présidence par les représentants des États membres pour une durée d'au moins un an. Cette solution avait toutefois laissé beaucoup de questions sans réponse ${ }^{24}$.

Article IV-444.

21 Article $23 \mathrm{du}$ projet de Constitution de la Convention. Cf. sur ce thème F. DEHOUSSE et W. COUSSENS, «Le Traité Constitutionnel de la Convention pour l'Europe: un nouveau pas pour l'intégration européenne?", op. cit., n 2.3.2.1.

22 Article I-24.

23 Cf. F. DeHousse et W. Coussens, « Le Traité Constitutionnel de la Convention pour l'Europe: un nouveau pas pour l'intégration européenne? ", op. cit., $\mathrm{n}^{\circ}$ 2.3.2.2.

24 Pour une évaluation de cette question et une analyse des résultats de la Convention à cet égard, F. DeHOuSSE et W. COUSSENS, "Which presidency for the future European Union? The Convention's dilemmas », in F. DEHOUSSE et W. COUSSENS (eds.), The Convention's Constitutional Treaty: old wine in a new bottle?, op. cit. 
De manière remarquable, quand on songe à toutes les déclarations des dernières années sur l'obsolescence de la présidence tournante, la CIG a finalement décidé de retourner largement à la situation actuelle ${ }^{25}$. La CIG consacre donc le statu quo, mais en y ajoutant les difficultés liées à une présidence collégiale. Cela ressemble ainsi au maintien d'un régime de plus en plus inefficace et rendu davantage complexe. Les modalités de ce nouveau régime n'ont pas été définies dans le texte même du traité, mais dans un projet de décision du Conseil européen annexé au traité ${ }^{26}$. Le Conseil européen doit encore adopter formellement cette décision, à la majorité qualifiée. Selon ce texte, la présidence du Conseil sera confiée à des groupes pré-établis de trois États membres et ceci pour une durée de 18 mois. Ces groupes seront composés sur une base de rotation égale parmi les États membres, tenant compte de leur diversité et de l'équilibre géographique au sein de l'Union. Chaque membre du groupe présidera à tour de rôle pendant une période de six mois toutes les formations du Conseil, à l'exception du Conseil des Affaires étrangères qui sera, comme cela avait déjà été prévu par la Convention, présidé par le ministre des Affaires étrangères. Il faut aussi noter que l'Eurogroupe aura quant à lui toujours la possibilité d'élire sa présidence propre.

Le texte présente un autre inconvénient, dans la mesure où il pourrait diminuer la capacité d'initiative de la Commission. Il prévoit en effet que les autres membres du groupe doivent aider la présidence dans toutes ses responsabilités sur la base d'un programme commun. Par ailleurs, le Conseil des Affaires générales doit assurer la cohérence et la continuité dans le travail des différentes formations dans le cadre de programmes pluriannuels en coopération avec la Commission. Le risque existe ainsi que ces dispositions sapent les prérogatives de la Commission en termes de programmation pluriannuelle (l'article I-26), menant alors à un conflit de programmes.

Ces éléments confirment l'impossibilité de préserver coûte que coûte le système actuel de la présidence tournante. Celui-ci ne peut en effet pas être préservé sans collectiviser l'exercice de la présidence entre plusieurs États membres. Or une telle formule ne fait, comme on vient de le voir, qu'ajouter plus de complexité et moins de visibilité à ce système. Elle implique en outre une coordination du travail de l'Union non pas seulement entre les États membres formant le groupe de la présidence, mais également avec la Commission, le ministre des Affaires étrangères et probablement avec le président du Conseil européen, sans oublier le président de l'Eurogroupe. Ce tissu complexe de lignes de coordination sera extrêmement difficile à gérer.

\subsection{LA COMMISSION}

\subsubsection{La composition de la Commission}

Selon le Traité de Nice, dès 2005, la Commission ne pourra plus comprendre qu'un représentant par État membre, les grands États perdant ainsi leur deuxième siège. De

25 Article I-24 $\int 7$.

26 CIG 87/04 ADD 2, Déclaration ad article I-24, paragraphe 7, concernant la décision du Conseil européen relative à l'exercice de la présidence du Conseil. 
plus, dès que l'Union aura atteint 27 membres, la Commission devra compter moins de commissaires que d'États membres. Les places devront être réparties entre ceux-ci selon un système de rotation égale encore à définir.

La Convention avait adopté à propos de la composition de la Commission un compromis complexe, selon lequel, dès 2009, la Commission comprendrait un représentant par État membre mais selon un système à deux niveaux. Ainsi le collège serait composé du président, du ministre des Affaires étrangères et de 13 « commissaires européens » nommés d'après un système de rotation égale entre l'ensemble des États membres. Les États membres n'ayant pas obtenu de «commissaire européen » auraient eu en compensation un «commissaire». Ce dernier ne détenait pas de droit de vote au sein de la Commission et n'appartenait pas au Collège ${ }^{27}$.

Le compromis constituait un recul par rapport à Nice. Le maintien d'une si grande Commission faisait en outre courir le risque que le partage de compétences au sein du Collège fasse apparaître des départements chargés de compétences quasi microscopiques. Il aurait par ailleurs pu affecter négativement la collégialité au sein de la Commission. Enfin, le rôle des commissaires non votants était resté plutôt ambigu.

La CIG a par conséquent abandonné cette idée d'une Commission à deux étages. Elle a opté au contraire pour une Commission réduite, mais seulement à partir de $2014^{28}$. Jusqu'en 2014, la Commission devrait comprendre un commissaire par État membre. Après 2014, le nombre de membres de la Commission, y compris le président et le ministre des Affaires étrangères, devra correspondre aux deux tiers du nombre d'États membres. Le Conseil européen pourra toutefois changer cette composition par un vote unanime. Bien que ce système soit positif en principe, on ne peut que regretter le fait que la CIG ait postposé dans le temps la limitation du nombre de commissaires. Un tel report risque en effet de paralyser le fonctionnement de la Commission pendant la décennie à venir. Il est dès lors difficile de trouver un motif qui puisse raisonnablement le justifier.

La CIG a par ailleurs ajouté une déclaration à la Constitution. Selon ce texte, lorsque la Commission n'inclura plus de ressortissants de tous les États membres, elle devra accorder une attention particulière à la nécessité de garantir la transparence absolue dans ses relations avec l'ensemble des États membres. En conséquence, la Commission devra rester en contact étroit avec tous les États membres et, à cet égard, accorder une attention particulière à la nécessité de partager les informations avec tous les États membres et de les consulter ${ }^{29}$. Il faut noter que les États membres seront traités sur un pied strictement égal en ce qui concerne la détermination de l'ordre et le temps passé par leurs ressortissants au sein de la Commission ${ }^{30}$.

\footnotetext{
Article I-25 du projet de Constitution de la Convention.

Article I-26 $\int 6$.

CIG 87/04 ADD 2, Déclaration ad article I-26.

Article I-26 \6.
} 


\subsubsection{La nomination de la Commission}

La CIG formalise quelque peu ha procédure relative à la nomination du président de la Commission. Elle reprend ainsi le texte de la Convention qui prévoyait que le Conseil européen nomme, en tenant compte des élections au Parlement européen, un candidat, que le Parlement européen «élit » par la suite ${ }^{31}$. Sans doute cela est-il assez proche de la situation actuelle. Il n'empêche que la référence aux élections européennes ouvrira peutêtre quelques perspectives à l'avenir pour une véritable élection de la Commission par le Parlement européen ${ }^{32}$.

Deux déclarations sont en outre adjointes à la Constitution en la matière. Selon la première $^{33}$, préalablement à la décision du Conseil européen, des représentants du Parlement européen et du Conseil européen procèdent aux consultations nécessaires dans le cadre jugé le plus approprié. Ces consultations porteront sur le profil des candidats aux fonctions de président de la Commission en tenant compte de l'élection au Parlement européen. Les modalités de ces consultations pourront être précisées, en temps utile, d'un commun accord entre le Parlement européen et le Conseil européen.

Selon la seconde déclaration ${ }^{34}$, lors du choix des personnes à qui l'ont fait appel pour remplir les fonctions de président du Conseil européen, de président de la Commission et de ministre des Affaires étrangères, il doit être tenu compte de la nécessité de respecter la diversité géographique et démographique de l'Union et de ses États membres ${ }^{35}$.

Il faut enfin regretter la limitation des prérogatives du président de la Commission quant à la désignation des différents commissaires. Dans le projet de la Convention, les États membres devaient lui présenter une liste de trois candidats, au sein de laquelle il était libre de choisir. La CIG a opté, elle, pour le statu quo, selon lequel chaque État membre peut faire «une suggestion» au président ${ }^{36}$, renforçant de la sorte la dépendance nationale des membres de la Commission vis-à-vis de leur niveau politique national.

\subsection{LE PARLEMENT EUROPÉEN}

Le nombre de membres du Parlement européen a augmenté lors de chaque élargissement de l'Union. Cette tendance ne peut que mener à terme à une tension entre d'une part la nécessité d'assurer une représentation appropriée des peuples des États membres et d'autre part la nécessité de limiter le nombre maximal des membres afin de garantir le fonctionnement efficace de l'institution.

31 Article I-27.

32 Pour une évaluation de cette question et une analyse des résultats de la Convention à cet égard, F. DEHOUSSE et W. COUSSENS, "Which presidency for the future European Union? The Convention's dilemmas », in F. DEHOUSSE et W. COUSSENS (réd.), The Convention's Constitutional Treaty: old wine in a new bottle?, op. cit.

33 CIG 87/04 ADD 2, Déclaration ad article I-27.

34 CIG 87/04 ADD 2, Déclaration ad articles I-22, I-27 et I-28.

35 Le Conseil européen n'a pas pris cette exigen ce «de diversité géographique» trop au sérieux lorsqu'il a choisi, quelques jours après le sommet de Bruxelles, M. Barroso comme candidat à la présidence de la Commission 2005-2008 et M. Solana comme futur ministre des Affaires étrangères.

36 Article I-27 \ 2. 
Pendant les négociations du Traité de Nice, les États membres avaient eu des difficultés à résoudre ce dilemme. Au lieu de poser un critère objectif, ils s'étaient alors embarqués dans un marchandage du nombre de sièges tout en laissant tomber le seuil maximal de membres, alors fixé à 700 membres depuis Amsterdam.

Dans le texte de la Convention, le résultat de Nice fut confirmé pour la période s'écoulant jusqu'aux élections européennes de 2009. À cette date et préalablement aux élections, un nouveau système aurait dû être décidé par le Conseil européen sur la base d'une proposition du Parlement européen. Celui-ci aurait dû être dégressivement proportionnel et aurait accordé au moins quatre sièges par État membre, le nombre maximal de membres du Parlement européen ayant été fixé à 736.

La CIG, quant à elle, a tout d'abord encore relevé le nombre maximal de parlementaires européens pour atteindre le nombre de 750. La CIG a par ailleurs relevé le nombre minimum de parlementaires par État membre (six au lieu de quatre) et fixé un maximum au nombre de sièges par État membre (96), ceci devant mener à une réduction du nombre actuel de représentants allemands ${ }^{37}$.

\subsection{LE MINISTRE DES AFFAIRES ÉTRANGÈRES}

La Convention proposa de joindre, dans un même poste, celui de «ministre des Affaires étrangères», deux fonctions actuellement exercées par deux personnes différentes: le haut représentant pour le PESC (HR) et le commissaire aux Relations extérieures. Cette double casquette a pour but d'en finir avec les conflits de compétence et les difficultés de gestion et de clarifier la voix de l'Europe sur la scène internationale.

Il y avait toutefois dans le projet établi par la Convention un peu de confusion quant à savoir si le ministre des Affaires étrangères devait démissionner de sa fonction de représentant PESC en cas de demande de sa démission par le président de la Commission ou de motion de censure du Parlement européen. Le Traité constitutionnel clarifie les conséquences de ces deux situations. Dans les deux cas, le ministre conservera en effet ses fonctions en matière PESC aussi longtemps que le Conseil européen n'aura pas décidé de mettre un terme à celles $-\mathrm{ci}^{38}$. En effet, seul ce dernier, agissant à la majorité qualifiée et avec l'accord du président de la Commission, a la possibilité de le sanctionner quant à ces fonctions ${ }^{39}$. Cette réponse de la CIG porte toutefois en elle le germe d'un conflit institutionnel entre, d'une part, le président de Commission et le Parlement européen et, d'autre part, le Conseil.

37 Article I-20.

Article I-26 \8, I-27 \3, I-28 \1 et III-340. Le « ministre » conservera en outre également, en cas de demande de démission de la part du président de la Commission, ses fonctions au sein de la Commission aussi longtemps que le Conseil européen n'aura pas décidé, en accord avec le président de la Commission, de le remplacer.

39 Article I-28 \ 1. 


\subsection{LA COOPÉRATION RENFORCÉE}

La Convention avait réussi à simplifier la matière des coopérations renforcées, en réduisant à deux le nombre de procédures : l'une pour la Politique commune de sécurité et de défense et l'autre pour les autres matières. Elle avait également renforcé leur adaptabilité en permettant aux États membres participants de modifier les règles de procédure décisionnelle applicables, permettant ainsi un passage à la majorité qualifiée là où l'unanimité est la règle. Elle avait enfin élargi le champ d'application de ces coopérations en ouvrant la possibilité de coopérations renforcées en matière de défense et en ne les limitant plus, en matière de PESC, à la simple mise en œuvre d'actions ou de positions communes.

La CIG s'est montrée plus timorée dans ce domaine. Elle a par exemple changé les règles de procédure décisionnelle dans les domaines de la PESC et de la défense. C'est ainsi désormais à l'unanimité que le Conseil doit décider d'autoriser une coopération renforcée dans ces deux domaines ${ }^{40}$. Il en est de même des décisions permettant par la suite à d'autres États membres de participer à cette coopération ${ }^{41}$. Cette modification de la CIG est un pas en arrière par rapport à Nice. La clause passerelle particulière qui permet au Conseil de changer la procédure décisionnelle est par ailleurs désormais exclue lorsqu'il s'agit de passer à un vote à la majorité qualifiée au sein d'une coopération renforcée ayant des implications militaires ou en matière de défense ${ }^{42}$. Enfin, une déclaration a été jointe à la Constitution. Selon celle-ci, les États membres ont la possibilité d'indiquer, lorsqu'ils font une demande tendant à établir une coopération renforcée, qu'ils ont déjà l'intention de se servir de cette clause ${ }^{43}$. Bien que cette possibilité soit présentée comme facultative, il semble évident que l'intention en est de limiter le recours à ces procédures.

Notons toutefois que la possibilité de coopération renforcée dans le secteur de la coopération judiciaire pénale a, elle, été accentuée (cf. 3.3.3).

\footnotetext{
40 Article III-419 2.

41 Article III-420 \$ 2.

42 Article III-422 $\int 3$.

${ }^{43}$ CIG 87/04 ADD 2, Déclaration ad article III-419.
} 


\section{LES POLITIQUES}

\subsection{LES DROITS FONDAMENTAUX ${ }^{44}$}

Les deux seules modifications introduites par la CIG, en matière de droits fondamentaux, concernent: (1) les explications relatives à la Charte et (2) l'adhésion à la Convention européenne de sauvegarde des Droits de l'homme et des libertés fondamentales $(\mathrm{CEDH})$.

\subsubsection{Les explications relative à la Charte}

Au moment de l'adoption de la Charte des droits fondamentaux en 2000, le Praesidium de la Convention rédigeant cette Charte avait élaboré des explications relatives à celle-ci au titre de conseils d'interprétation. Avec l'inclusion de la Charte dans le projet de Constitution, ces explications furent mises à jour par le Praesidium de la Convention européenne sur l'avenir de l'Europe ${ }^{45}$. Le Préambule de la Partie II du projet, ne mentionne toutefois pas ces modifications et se contente de déclarer que «la Charte sera interprétée par les juridictions de l'Union et des États membres en prenant dûment en considération les explications établies sous l'autorité du Praesidium de la Convention qui a élaboré la Charte ».

La CIG a, d'une certaine manière, comblé cette lacune. Elle a ajouté une clause au susdit paragraphe se référant cette fois à la mise à jour faite sous la responsabilité du Praesidium de la Convention européenne. Elle a également ajouté un nouveau paragraphe à l'article II-112, selon lequel «les explications élaborées en vue de guider l'interprétation de la Charte des droits fondamentaux sont dûment prises en considération par les juridictions de l'Union et des États membres ». Une déclaration doit enfin être incorporée à l'Acte final, selon laquelle l'Union déclare: "La Conférence prend note des explications relatives à la Charte des droits fondamentaux, établies sous l'autorité du Praesidium de la Convention qui a élaboré la Charte et mises à jour sous la responsabilité du Praesidium de la Convention européenne.»

La raison principale du zèle avec lequel il est fait référence à ces explications réside dans la crainte britannique de voir l'inclusion de la Charte dans la Constitution étendre les compétences de l'Union et «créer» ainsi des droits nouveaux inexistants dans l'ordre juridique britannique.

Bien que les explications puissent être perçues comme une barrière à une interprétation dynamique de la Charte, leur mention dans le traité n'est pas nécessairement un développement négatif. Il ne doit en effet pas être oublié que celles-ci n'ont pas le statut

44 Pour une évaluation des résultats de la Convention à cet égard, cf. F. DEHOUSSE et J. GARCIA, «The issue of fundamental rights in the European Convention », in F. DEHOUSSE et W. COUSSENS (réd.), The Convention's Constitutional Treaty: old wine in a new bottle?, op. cit.

45 CONV 828/1/03. 
de loi et qu'elles ne sont qu'un outil d'interprétation parmi d'autres. De plus, la Cour aurait de toute manière utilisé ces explications lors de son interprétation de la Charte, qu'elles aient été mentionnées dans la Constitution ou non.

\subsubsection{L'adhésion à la CEDH}

La CIG présente quelques modifications positives quant à l'adhésion de l'Union à la CEDH. D'abord, celle-ci est rendue obligatoire pour l'Union. La Constitution déclare en effet désormais que l'Union "adhère » et non plus qu'elle "cherche à adhérer ${ }^{46}$ ». Ensuite, l'adhésion est facilitée par l'abandon de l'exigence de l'unanimité au sein du Conseil au profit d'un vote à la majorité qualifiée lors de la conclusion de cette Convention ${ }^{47}$. Enfin, un protocole a été annexé à la Constitution afin de définir la position que défendra l'Union lors des négociations d'adhésion.

\subsection{L'UNION ÉCONOMIQUE ET MONÉTAIRE ${ }^{48}$}

\subsubsection{Le pilier monétaire}

La CIG a fait quelques modifications au «pilier monétaire ». D'abord, elle a ajouté la stabilité des prix comme objectif de l'Union dans la première partie de la Constitution ${ }^{49}$. Elle a ensuite prévu que le Conseil européen nomme les membres du directoire à la majorité qualifiée ${ }^{50}$. Enfin, elle a écarté au profit de l'unanimité le recours à la majorité qualifiée lors de l'adoption de la loi qui confie à la Banque centrale européenne des missions spécifiques ayant trait aux politiques en matière de contrôle prudentiel des établissements de crédit et autres établissements financiers, à l'exception des entreprises d'assurances. À cette occasion, le Parlement n'est en outre que consulté ${ }^{51}$.

\subsubsection{La reconnaissance de la spécificité de la zone euro dans le processus décisionnel du Conseil}

Le texte adopté par la CIG permet une meilleure reconnaissance de la spécificité de la zone euro dans le processus décisionnel Ecofin. Une telle évolution était nécessaire si l’on désire éviter que les États qui ne sont pas membres de la zone euro empêchent le processus décisionnel d'être efficace dans les domaines où les membres de la zone ont des intérêts spécifiques.

\footnotetext{
46 Article I-9 $\$ 2$.

47 Article III-325 $\$ 8$.

48 Pour une évaluation des résultats de la Convention à cet égard, cf. F. DEHOUSSE et W. COUSSENS, «Economic and Monetary Union the Convention », in F. DEHOUSSE et W. COUSSENS (réd.), The Convention's Constitutional Treaty: old wine in a new bottle?, op. cit.

49 Article I-3 $\int 3$.

50 Article III-382 \ 2.

51 Article III-185 \ 6.
} 
La Convention était restée proche du statu quo à cet égard. Elle s'était en effet contentée de prévoir la possibilité, pour les États membres qui font partie de la zone euro, d'adopter de manière autonome des mesures spécifiques concernant la coordination économique et budgétaire ${ }^{52}$. Elle avait également prévu un protocole reconnaissant le rôle de l'Eurogroupe en tant que forum de discussion informel, présidé par un président choisi pour deux ans et demi parmi ses membres ${ }^{53}$.

La CIG est allée plus loin. La Constitution stipule en outre désormais que les droits de vote des États qui ne font pas partie de la zone euro sont en effet suspendus lors de l'adoption par le Conseil d'une part des recommandations faites à des États dont la monnaie est l'euro dans le cadre de la surveillance multilatérale et d'autre part des mesures relatives aux déficits excessifs concernant les États membres dont la monnaie est l'euro ${ }^{54}$. Cela signifie que, par exemple, seuls les États membres de la zone euro pourront voter lors de décisions telles que l'envoi d'un premier avertissement à un membre de la zone euro dont la politique économique risque de compromettre le bon fonctionnement de l'UEM, la constatation de l'existence d'un déficit excessif dans un État faisant partie de la zone ou la définition des mesures correctrices à prendre par cet État.

Cette évolution constitue un réel progrès au vu du nombre important de pays ne participant pas à l'euro depuis l'élargissement. Il ne s'agit toutefois pas de la panacée : les États membres de la zone euro n'ont en effet jusqu'ici pas démontré plus d'inclination à mettre leurs pairs sous pression que les États hors de la zone, comme l'a démontré leur piètre prestation dans la tâche de faire respecter les procédures de déficit excessif du Traité de Maastricht complétées par le Pacte de stabilité et de croissance de 1997.

Un autre changement notable, dans ce cadre, tient au fait que l'entrée de nouveaux membres dans la zone euro exigera désormais une recommandation des États membres de cette zone à propos de cette accession. Cette recommandation devra être adoptée dans les six mois de la réception par le Conseil de la proposition de la Commission en cette matière ${ }^{55}$. La décision finale sera toutefois toujours prise par le Conseil dans son intégralité. C'est néanmoins un changement bienvenu, puisqu'il permet un contrôle supplémentaire quant à l'accession de nouveaux membres, assurant de la sorte que seuls les États membres remplissant les conditions exigées peuvent adhérer à l'euro. Étant donné le nombre important d'États membres en dehors de la zone euro dans le Conseil, il y existe toutefois un risque que ceux-ci ne s'engagent dans un exercice de marchandage sur cette question pourtant importante.

\subsubsection{Les prérogatives de la Commission dans la procédure de déficit excessif}

Le texte adopté par la Convention avait permis un renforcement limité des pouvoirs de la Commission en ce qui concerne la procédure de déficit excessif. Plus particulièrement, la Commission faisait non plus une recommandation mais une proposition au Conseil

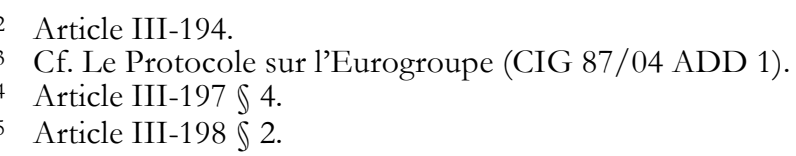


lorsque celui-ci doit prendre position sur l'existence d'un déficit ou sur les mesures correctrices à prendre par l'État membre en cause afin de mettre fin à son déficit excessif. Cette modification aurait assurément renforcé le rôle de la Commission, puisque, à moins d'une unanimité au sein du Conseil, l'accord de la Communication serait devenu nécessaire pour amender cette proposition.

La CIG est partiellement revenue sur ce progrès. L'exigence d'une proposition de la Commission n'est en effet plus maintenue que pour les décisions relatives à l'existence d'un déficit excessif. Pour ce qui est des mesures correctrices, la CIG est revenue à la simple exigence d'une recommandation ${ }^{56}$.

De ce fait, la CIG a fait marche arrière sur l'élément le plus important du progrès de la Convention à cet égard. En effet, l'importance que peut avoir le passage d'une recommandation à une proposition pour ce qui est de l'influence de la Commission dépend surtout du fait de savoir si le contenu de la décision en cause est modifiable et ne se résume pas simplement à un oui ou à un non ${ }^{57}$. Dès lors le progrès de la Convention présentait surtout de l'importance en matière de mesures correctrices et c'est malheureusement sur celles-ci que la CIG est revenue.

\subsection{LA SÉCURITÉ INTÉRIEURE}

Les améliorations réalisées dans le fonctionnement de l'Espace de liberté, de sécurité et de justice proposées par la Convention européenne ont sans doute constitué l'un des principaux accomplissements de cette dernière. Le vote à la majorité qualifiée et la codécision ont été étendus à la plupart des matières. Eurojust et Europol ont vu leurs pouvoirs augmenter. Une nouvelle figure, le procureur européen, a été envisagée ${ }^{58}$.

La plupart des réalisations de la Convention dans le secteur Justice-Affaires intérieu res ont été maintenues par la CIG. Les propositions de la Convention sur l'asile, l'immigration et les questions liées au contrôle des frontières l'ont également été, créant de la sorte une base à de véritables politiques communes dans ces secteurs. Les articles proposés par la Convention en matière de coopération policière n'ont pas non plus été modifiés. En revanche, le texte final concernant le secteur de la coopération judiciaire pénale est, quant à lui, sous plusieurs aspects, moins ambitieux que le texte de la Convention.

6 Artide III-184 \ 6.

57 F. AMTENBRINK et J. DE HAAN, «Economic governance in the European Union : fiscal policy discipline versus flexibility ", Common Market Law Review, 2003, pp. 1075-1106.

58 Pour une évaluation de cette question et une analyse des résultats de la Convention à cet égard, cf. F. Dehousse et J. GARCIA, The Area of Freedom, Security and Freedom in the European Convention, in F. DeHOUSSE et W. COUSSENS (réd.), The Convention's Constitutional Treaty: old wine in a new bottle?, op. cit. 


\subsubsection{Eurojust}

Le texte de la Convention avait considérablement renforcé le rôle d'Eurojust. Selon sa décision de base ${ }^{59}$, Eurojust ne peut que demander aux autorités compétentes d'entreprendre une enquête ou une poursuite sur des actes spécifiques. En revanche, le texte de la Convention attribuait directement à Eurojust le droit de déclencher et de coordonner des poursuites pénales. La CIG est toutefois revenue de façon partielle sur cette évolution. La Constitution déclare en effet désormais qu'une des tâches d'Eurojust est le déclenchement d'enquêtes pénales ainsi que la proposition de déclenchement de poursuites conduites par les autorités nationales compétentes ${ }^{60}$. Eurojust perd ainsi le droit d'initier les poursuites pour ne garder que celui d'initier des enquêtes. Le texte CIG (art III-273) tout en étant une amélioration par rapport à la situation actuelle (où Eurojust ne peut que «demander» d'entreprendre des enquêtes et des poursuites), ne constitue pas moins une marche arrière par rapport au texte de la Convention, qui attribuait à Eurojust le pouvoir d'initier des poursuites et des enquêtes.

\subsubsection{Le procureur européen}

L'article III-175 du texte de la Convention prévoyait la création d'un procureur européen, compétent pour combattre la criminalité grave ayant une dimension transfrontière ainsi que les infractions portant atteinte aux intérêts de l'Union. Le texte final de la CIG limite cette compétence. Désormais, le procureur n'est plus compétent que pour protéger les intérêts financiers de l'Union. Une possibilité d'étendre, par une décision unanime, la compétence du procureur à d'autres crimes est toutefois prévue. Cette extension pourrait probablement aller de pair avec la décision (unanime) de création du procureur européen ${ }^{61}$.

\subsubsection{Règles communes minimales dans le secteur du droit pénal}

Les articles III-171 et III-172 du texte de la Convention prévoyaient l'établissement de règles communes minimales dans le secteur du droit pénal en ce qui concerne respectivement les questions procédurales et la définition des délits et des sanctions. Les deux articles contenaient ainsi une liste de questions à traiter suivant la procédure législative normale (majorité qualifiée dans le Conseil et co-décision avec le Parlement européen) et la possibilité d'y ajouter d'autres questions, mais seulement après l'adoption d'une décision unanime par le Conseil.

Le texte de la CIG maintient les dispositions du texte de la Convention ${ }^{62}$, mais ajoute, dans les deux articles, une procédure spéciale, la procédure de frein/accélérateur. Le «frein» concerne la possibilité donnée à n'importe quel État membre de bloquer l'adoption d'une mesure dès lors qu'il estime qu'une telle mesure porterait atteinte aux

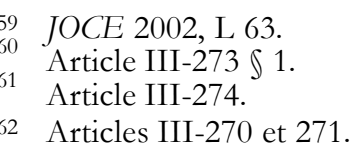


aspects fondamentaux de son système juridique. Dans une telle éventualité, il peut alors demander que le Conseil européen soit saisi du projet de loi-cadre, celui-ci ayant alors le choix, dans les quatre mois suivant la suspension de la procédure, soit de renvoyer le projet de loi-cadre au Conseil, mettant ainsi fin à la suspension de la procédure, soit de demander à la Commission ou au groupe d'États membres dont le projet de loi-cadre émane de soumettre un nouveau projet.

L' «accélérateur» concerne le droit attribué aux États membres souhaitant adopter une telle mesure par la voie d'une coopération renforcée de le faire. Si aucune action n'a en effet été prise par le Conseil européen dans les quatre mois prévus, ou si dans un délai de douze mois à compter de la présentation d'un nouveau projet de loi-cadre européenne, celle-ci n'a toujours pas été adoptée, les États membres qui veulent établir une coopération renforcée sur la base du projet de loi-cadre concerné informent le Parlement européen, le Conseil et la Commission de leur intention. Dans un tel cas et pourvu quau moins un tiers des États membres fasse cette demande, l'autorisation de procéder à la coopération renforcée est réputée accordée. Ils sont alors dispensés de la nécessité d'une décision d'autorisation (par majorité qualifiée) du Conseil mais également de l'exigence d'une proposition de la Commission et du consentement du Parlement européen.

Cette réforme est ambiguë. D'une part, elle constitue un pas en arrière par rapport à la Convention. Même si le champ d'application de la majorité qualifiée reste en principe le même que dans le texte de la Convention, la nouvelle procédure ralentira très certainement le processus législatif et le contenu des propositions sera probablement dilué pour gagner l'accord de tous les États membres et éviter le recours à des coopérations renforcées. D'autre part, face à une opposition insurmontable d'un État membre, l'élimination de quelques étapes dans la procédure offre une simplification indéniable pour les États membres souhaitant aller de l'avant et pourrait dès lors faciliter l'établissement de coopérations intéressantes et fructueuses.

\subsubsection{Le protocole sur la position du Royaume-Uni et de l'Irlande}

Le texte de la Convention n'avait pas inclus le protocole sur la position du Royaume-Uni et de l'Irlande, qui exclut la participation du Royaume-Uni et de l'Irlande dans les mesures liées à l'immigration, à l'asile, aux contrôles aux frontières et au droit civil. Le texte final de la CIG maintient, quant à lui, expressément le protocole, mais, étonnamment, il élargit la portée des exceptions accordées à ces deux pays. Il prévoit en effet désormais qu'en principe, le Royaume-Uni et l'Irlande ne participent point à l'adoption et à l'application des mesures liées à un aspect important de la coopération policière, à savoir l'échange de renseignements. Pourtant, ils peuvent notifier au président du Conseil leur désir de participer à l'adoption et à l'application de telles mesures, à la suite de quoi ils y sont habilités ${ }^{63}$.

63 CIG 87/04 ADD 1, Protocole sur la position du Royaume-Uni et de l'Irlande à l'égard des politiques relatives aux contrôles aux frontières, à l'asile et à l'immigration, ainsi qu'à l'égard de la coopération judiciaire en matière civile et de la coopération policière. 
Il est difficile de comprendre pourquoi, au lieu de réduire ou d'éliminer la portée de ce protocole, le texte CIG l'a élargi pour couvrir un secteur auquel ces pays ont participé jusqu'ici de manière entière. L'explication donnée par le gouvernement britannique selon laquelle cette nouvelle clause a été incluse pour éviter l'harmonisation fiscale apparait plutôt peu convaincante.

\subsection{LES RELATIONS EXTÉRIEURES}

En ce qui concerne les relations extérieures de l'Union, les changements principaux par rapport au projet de la Convention ${ }^{64}$ concernent la coopération structurée permanente, la défense mutuelle, le processus décisionnel dans la politique commerciale commune, le service européen pour l'action extérieure et la compétence de la Cour de jJustice dans la PESC.

\subsubsection{La coopération structurée permanente}

Dans le projet de la Convention, la coopération structurée était une forme particulière de coopération renforcée dans le secteur PESC, établie sur base du traité par les États membres remplissant des critères de capacités militaires plus élevés et souhaitant entreprendre des engagements plus contraignants en vue des missions les plus exigeantes. Dans ce cadre, un protocole aurait donné la liste des États membres participants et aurait fixé les critères et les engagements. La coopération structurée aurait tout autant été capable d'effectuer des missions PESC que ses propres tâches ${ }^{65}$.

Le Traité constitutionnel change le nom de cette coopération, désormais appelée «coopération structurée permanente». Le protocole attaché au Traité constitutionnel n'aura par ailleurs plus pour tâche de lister les États membres participant à la coopération. Il énumèrera seulement les conditions que les États membres devront remplir pour y participer ${ }^{66}$.

Ces conditions sont tout d'abord que les États membres doivent s'engager à deux objectifs :

- procéder plus intensivement au développement de leurs capacités de défense, par le développement de leurs contributions nationales et la participation, le cas échéant, dans des forces multinationales, dans les principaux programmes européens d'équipement et dans l'activité de l'Agence européenne d'armements dans le domaine du développement des capacités de défense, de la recherche, de l'acquisition et de l'armement;

64 Pour une évaluation de cette question et une analyse des résultats de la Convention à cet égard, cf. F. DeHOUSSE, P. VAN DEN BRULE et T. ZGAJEWSKI, "The Union's external relations in the Convention », in F. Dehousse et W. Coussens (réd.), The Convention's Constitutional Treaty : old wine in a new bottle?, op. cit.

65 Articles I-40 \$6 et III-213 du projet de Constitution de la Convention.

66 CIG 87/04 ADD 1, Protocole sur la coopération structurée permanente établie par l'articles I-41, paragraphe 6 et par l'article III-312 de la Constitution. 
- avoir la capacité de fournir, au plus tard en 2007, soit à titre national, soit comme composante de groupes multinationaux de forces, des unités de combat ciblées pour les missions envisagées, configurées sur le plan tactique comme une formation de combat, avec les éléments de soutien, y compris le transport et la logistique, capables d'entreprendre, dans un délai de 5 à 30 jours, des missions de la politique de sécurité et de défense commune, et soutenables pour une période initiale de 30 jours, prorogeable jusqu'au moins 120 jours.

Pour réaliser ces objectifs, les États membres participant à la coopération devront s'engager à :

- coopérer, en vue d'atteindre des objectifs agréés concernant le niveau des dépenses d'investissement en matière d'équipements de défense et à réexaminer régulièrement ces objectifs, à la lumière de l'environnement de sécurité et des responsabilités internationales de l'Union ;

- rapprocher, dans la mesure du possible, leurs outils de défense, notamment en harmonisant l'identification des besoins militaires, en mettant en commun et, le cas échéant, en spécialisant leurs moyens et capacités de défense, ainsi qu'en encourageant la coopération dans les domaines de la formation et de la logistique;

- prendre des mesures concrètes pour renforcer la disponibilité, l'interopérabilité, la flexibilité et la déployabilité de leurs forces, notamment en identifiant des objectifs communs en matière de projection de forces, y compris éventuellement en réexaminant leurs procédures décisionnelles nationales ;

- coopérer pour assurer qu'ils prennent les mesures nécessaires pour combler, y compris par des approches multinationales et sans préjudice des engagements les concernant au sein de l'OTAN, les lacunes constatées dans le cadre du « Mécanisme de développement des capacités »;

- participer, le cas échéant, au développement de programmes communs ou européens d'équipements majeurs dans le cadre de l'Agence.

Les États membres désirant participer à la coopération structurée permanente, qui remplissent les critères et souscrivent aux engagements en matières de capacités militaires repris au protocole, doivent notifier leurs intentions au Conseil et au ministre ${ }^{67}$. Dans les trois mois suivant une telle notification, le Conseil adoptera alors, après consultation du ministre, une décision européenne établissant la coopération et fixant la liste des États membres participants ${ }^{68}$.

Si à un stade ultérieur, un État membre souhaite participer à la coopération, il devra suivre la même procédure. Toutefois, dans ce contexte, seuls les États membres participant déjà à la coopération prendront part au vote ${ }^{69}$. Le texte de la CIG prévoit également une procédure de retrait et de suspension. Si un État membre participant ne remplit ainsi plus les critères ou ne peut plus assumer ses engagements, le Conseil peut suspendre sa participation. Seuls les États membres participants, à l'exception de l'État membre en question, prendront part à ce vote de suspension ${ }^{70}$. Au cas où un État

\footnotetext{
Article III-312 \& 1.

Article III-312 \& 2

Article III-312 $\$ 3$.

Article III-312 \ 4.
} 
membre participant veut se retirer de la coopération, il doit simplement notifier son intention au Conseil pour cesser sa participation ${ }^{71}$.

De manière remarquable, le texte de la CIG prévoit que le Conseil agit à la majorité qualifiée lorsqu'il établit la coopération structurée permanente, admet de nouveaux États membres dans la coopération ou suspend la participation d'un État membre à celle-ci. Il s'agit là d'une différence fondamentale par rapport au projet de la Convention, selon lequel la coopération structurée était établie par le traité, exigeant ainsi l'entente des représentants des gouvernements à la CIG et une ratification nationale unanime de tous les États membres, et où toutes les décisions étaient adoptées à l'unanimité ${ }^{72}$. Toutes les autres décisions dans le cadre de la coopération structurée permanente sont adoptées par un vote unanime des États membres participants ${ }^{73}$.

Contrairement au projet de la Convention, les États membres non participants ne sont par ailleurs plus exclus des débats du Conseil relatifs à la coopération structurée ${ }^{74}$. Le rôle du ministre des Affaires étrangères dans le processus décisionnel est en outre renforcé par rapport à ce qui était prévu dans le projet de la Convention. La négociation intergouvernementale a ainsi abouti à un nouvel équilibre, plus efficace et plus inclusif.

Finalement, il faut remarquer que le texte définitif ne stipule plus que le Conseil peut demander aux États membres participant à la coopération structurée d'effectuer une mission de la Politique de sécurité et de défense commune ${ }^{75}$. La coopération structurée semble dès lors devoir se limiter à n'être qu'une coopération flexible en matière de capacités, avec un rôle central toutefois attribué aux unités de combat. Il reste à voir quelle sera la valeur ajoutée d'une telle coopération ${ }^{76}$.

\subsubsection{La défense mutuelle}

Le projet de la Convention prévoyait qu'aussi longtemps que le Conseil n'aura pas décidé de passer à une défense commune, une coopération plus étroite serait instaurée, dans le cadre de l'Union, en matière de défense mutuelle. Cette coopération plus étroite aurait été ouverte à tous les États membres et une déclaration aurait énuméré les f́tats acceptant cet engagement de défense mutuelle. Un État membre participant à cette coopération qui aurait fait l'objet d'une agression armée sur son territoire était censé informer les autres États participants de la situation, ce qui lui permettait alors de demander l'aide et l'assistance de ceux-ci. Au titre de cette coopération, les États membres participants devaient travailler en étroite coopération avec l'OTAN. Cette coopération étroite ne devait pas affecter, pour les États membres qui sont concemés, les droits et les obligations résultant du Traité de l'Atlantique Nord ${ }^{77}$. Le texte de la Convention contenait également un engagement limité de l'Union et de tous les États

71 Article III-312 \5.

72 Article III-201 $\int 1$ du projet de Constitution de la Convention.

73 Article III-312 \& 6.

74 Article III-312 \6 de la Constitution comparé à l'article III-213 \3 du projet de Constitution de la Convention.

75 Article III-213 \ 4 du projet de Constitution de la Convention.

76 S. BISCOP, Able and willing? Assessing the EU capacity for military action (à paraitre).

77 Articles I-40 \ 7 et III-214 du projet de Constitution de la Convention. 
membres d'aider tout État membre victime d'une attaque terroriste ou d'un désastre naturel ou provoqué par l'homme (la clause de solidarité) ${ }^{78}$.

Le Traité constitutionnel maintient la clause de solidarité ${ }^{79}$, mais modifie radicalement la coopération plus étroite sur la défense mutuelle. L'article III-214 du projet de Constitution de la Convention est supprimé et l'article $\mathrm{F} 40$ \& 7 complètement modifié devient l'article I-41 \ 7 dans le texte de la CIG. En vertu du nouvel article I-41 \$7, tous les États membres, y compris les neutres, prennent ainsi l'engagement, en cas d'agression armée sur le territoire d'un autre État membre, d'apporter à la victime aide et assistance par tous les moyens en leurs pouvoirs ( $y$ compris les moyens militaires), et ceci conformément à la Charte des Nations unies. La défense collective n'est donc désormais plus une coopération plus étroite entre États membres désirant entrer dans un tel engagement, mais une obligation résultant de l'article 51 de la Charte ONU.

Le Traité constitutionnel déclare cependant d'une part, que cette obligation n'affecte pas le caractère spécifique de la politique de sécurité et de défense de certains États membres, par exemple les neutres, et que, d'autre part, les engagements et la coopération dans ce secteur demeurent conformes aux engagements souscrits au sein de l'Otan, qui reste, pour les États qui en sont membres, le fondement de leur défense collective et l'instance de sa mise en œuvre.

La portée de cet article est dès lors plus réduite qu'il n'y paraît. Le lien entre l'engagement de défense mutuelle et le caractère spécifique de la politique de sécurité et de défense de certains États membres, comme les neutres, n'est par ailleurs pas clair. S'il doit signifier que ces États membres peuvent décider de ne pas accomplir cet engagement à cause de leur neutralité, la portée de cet article est en effet alors encore plus réduite.

\subsubsection{La procédure de décision dans la politique commerciale commune}

Dans le projet de la Convention, l'exigence d'unanimité était maintenue pour la négociation et la conclusion d'accords dans les domaines du commerce des services impliquant des déplacements de personnes et des aspects commerciaux de la propriété intellectuelle dès lors que de tels accords incluaient des dispositions pour lesquelles l'unanimité est requise pour l'adoption de règles internes. De même, elle était nécessaire pour la négociation et la conclusion d'accords dans le domaine du commerce des services culturels et audiovisuels lorsque ceux-ci risquent de porter atteinte à la diversité culturelle et linguistique de l'Union (l'exception culturelle) ${ }^{80}$.

La CIG a allongé la susdite liste. Elle y a en effet inclus les accords dans le domaine des investissements étrangers directs et a supprimé l'exigence selon laquelle les accords dans le domaine des services doivent impliquer des mouvements de personnes pour devoir être conclus à l'unanimité ${ }^{81}$. Enfin, une «exception sociale» a été ajoutée à l'exception culturelle déjà présente dans le projet de la Convention. Celle-ci prévoit que dans le

78 Article I-42 du projet de Constitution de la Convention.

Article I-43.

80 Article III-217 \ 4 du projet de Constitution de la Convention.

81 Article III-315 \ 4. 
domaine du commerce des services sociaux, d'éducation et de santé, la négociation et la conclusion des accords internationaux a lieu à l'unanimité dès lors que ces derniers risquent de perturber gravement l'organisation de ces services au niveau national et de porter atteinte à la responsabilité des États membres pour la fourniture de ces services ${ }^{82}$. Il s'agit là d'un changement important, puisque le risque de perturbation grave est évidemment très difficile à évaluer.

\subsubsection{Le service européen pour l'action extérieure}

La déclaration sur la création d'un service européen pour l'action extérieure annexée au projet de Constitution de la Convention a été intégrée par la CIG dans l'article III-296. L'organisation et le fonctionnement de ce service seront établis par une décision européenne adoptée sur proposition du ministre après consultation du Parlement européen et après approbation de la Commission. Cette formulation limite ainsi quelque peu les pouvoirs de la Commission par rapport au projet de la Convention ${ }^{83}$.

\subsubsection{La compétence de la Cour de justice dans le domaine PESC}

Le nouvel article III-376 limite davantage les compétences de la Cour de justice dans le secteur de la PESC. Dans le projet de la Convention, la Cour était compétente pour l'intégralité des décisions européennes du Conseil européen concernant les intérêts stratégiques et les objectifs de l'Union. La CIG recule sur ce point. La Constitution adoptée par la CIG prévoit en effet désormais que la Cour n'a aucune compétence quant à ces décisions dans la mesure où celles-ci concernent la PESC ${ }^{84}$. Ces décisions ne pourront par conséquent être soumises au contrôle de la Cour que dans la mesure où elles concernent d'autres secteurs de l'action externe de l'Union.

\subsection{LA POLITIQUE SOCIALE}

\subsubsection{La clause sociale horizontale}

La Convention avait examiné la possibilité d'insérer dans le traité une clause de cohérence horizontale en matière de politique sociale comme il en existe déjà à l'heure actuelle en matière d'environnement et de défense du consommateur. Ces clauses imposent la prise en compte des exigences environnementales et de protection du consommateur dans la définition et la mise en œuvre des politiques et des activités de

Article III-315 $\$ 4$.

83 Cf. la déclaration sur la création d'un Service européen pour l'action extérieure annexée au projet de Constitution de la Convention qui exigeait un accord entre «le Conseil des ministres et la Commission, sans préjudice des droits du Parlement européen, pour établir sous l'autorité du ministre un service commun ».

84 Selon l'article III-376, la Cour de Justice n'a pas de compétence en ce qui concerne l'article III-293 dès lors que celui-ci concerne la PESC. 
l'Union. La Convention avait cependant échoué à intégrer une telle clause en matière sociale.

La CIG a toutefois, de manière inattendue, réussi là où la Convention avait auparavant échoué. Le nouvel article III-117 stipule désormais que, dans la définition et la mise en œuvre de ses politiques et actions, l'Union prendra en compte les exigences liées à la promotion d'un niveau d'emploi élevé, à la garantie d'une protection sociale adéquate, à la lutte contre l'exclusion sociale ainsi qu'à un niveau élevé d'éducation, de formation et de protection de la santé humaine. Il est cependant encore trop tôt pour savoir si cette clause rendra vraiment plus « sociales » les politiques de l'Union.

La CIG a ensuite inséré une référence à un «sommet social tripartite pour la croissance et l'emploi ». Ce sommet doit contribuer au dialogue social dans l'Union ${ }^{85}$.

\subsubsection{Le recul en matière de protection sociale des travailleurs migrants}

Sur un ton plus négatif, la CIG a fait marche arrière sur la question de la coordination des régimes de sécurité sociale pour les travailleurs migrants. Selon la Convention, les décisions en cette matière devaient dorénavant être prises à la majorité qualifiée au sein du Conseil. La CIG a modifié cela. Elle prévoit désormais la possibilité pour un État membre, qui estime qu'un projet de loi ou de loi-cadre en cette matière porterait atteinte à des aspects fondamentaux de son système de sécurité sociale ou en affecterait l'équilibre financier, de demander que le Conseil européen soit saisi de la question, ce qui suspend ainsi la procédure de vote à la majorité qualifiée. Dans un délai de quatre mois à compter de cette suspension, le Conseil européen doit alors soit renvoyer le projet au Conseil, soit demander à la Commission de soumettre un nouveau projet. Cela revient dès lors à réintroduire d'une certaine manière l'unanimité dans cette matière ${ }^{86}$.

La CIG a aussi proposé une procédure assez semblable à celle prévue dans le secteur de la coopération judiciaire pénale. Il n'y a cependant aucune référence à une éventuelle autorisation automatique pour une coopération renforcée dans ce domaine. Ainsi, alors que dans le secteur de la coopération judiciaire pénale une procédure de "freinaccélérateur » a été prévue, la CIG n'a prévu qu'un «frein» pour le régime de sécurité sociale des travailleurs migrants.

\subsubsection{Les services d'intérêt économique général}

La CIG, tout comme la Convention l'avait fait, a également établi une base légale à propos des services d'intérêt économique général, stipulant à ce propos que l'Union et les États membres veillent à ce que ces services fonctionnent sur la base de principes et de conditions qui leur permettent d'accomplir kurs missions. Une loi européenne doit définir ces principes et conditions ${ }^{87}$. La CIG précise que cette loi sera établie sans

\footnotetext{
Article I-48,

Article III-136.

Article III-122.
} 
préjudice de la compétence qu'ont les États membres de fournir, de faire exécuter et de financer de tels services.

Un dernier point remarquable en la matière réside dans la déclaration annexée à la Constitution selon laquelle la politique sociale appartient essentiellement à la compétence des États membres ${ }^{88}$. Les mesures d'encouragement et de coordination à prendre au niveau de l'Union doivent dès lors revêtir un caractère complémentaire. Elles servent à renforcer la coopération entre États membres et non pas à harmoniser des systèmes nationaux. Elle ajoute toutefois que cette précision est sans préjudice des dispositions de la Constitution attribuant des compétences à l'Union. Pourtant, cette déclaration oblige à se poser des questions quant à la nature des compétences de l'Union dans le domaine social, que la Convention avait qualifié de domaine de compétence partagée. La déclaration limite quelque peu la nature partagée de cette compétence, réduisant aussi la clarté de la distribution des compétences posée par la Constitution. Une telle clarté constituait pourtant l'un des objectifs principaux poursuivis par cet exercice de « constitutionnalisation ».

\subsection{LA FISCALITÉ}

Le projet de la Convention n'avait réalisé aucun progrès significatif en termes de politique fiscale. L'exigence d'unanimité avait ainsi été maintenue en matière de fiscalité indirecte, de fiscalité des sociétés et de taxes environnementales. La seule très petite avancée en matière de vote à la majorité qualifiée qui avait été acceptée en matière de fiscalité indirecte et de sociétés portait sur la possibilité donnée au Conseil de décider à l'unanimité de passer à un vote à la majorité qualifiée pour ce qui concerne certaines mesures fiscales liées à la coopération administrative et à la lutte contre la fraude et l'évasion fiscale ${ }^{89}$.

La CIG a mis fin à ce petit progrès. Elle a tout simplement éliminé les dispositions insérées par la Convention. Ce revirement pourrait toutefois avoir un impact négatif sur le fonctionnement du marché intérieur. Les sociétés multinationales implantées en Europe sont en effet confrontées à une forêt opaque de 25 régimes différents de taxation. Ces différences sont un obstacle important à la libre circulation des capitaux, des services et des travailleurs, et donc au fonctionnement du marché commun. Il n'est évidemment pas question de définir un système unique européen de fiscalité. Mais une harmonisation limitée des systèmes fiscaux aurait pu cependant offrir une plus grande simplicité et une meilleure efficacité pour les sociétés implantées dans l'Union, améliorant de la sorte le fonctionnement du marché unique. Dans une Union élargie, le passage au vote à la majorité qualifiée est d'autant plus nécessaire.

88 CIG 87/04 ADD 2, Déclaration ad article III-213.

89 Articles III-62 et III-63 du projet de Constitution de la Convention. 


\section{LE BUDGET}

\subsection{LES RESSOURCES PROPRES}

Selon le projet de Constitution établi par la Convention, la loi européenne fixant la limite des ressources propres et pouvant établir de nouvelles catégories de ressources continuait à devoir être adoptée à l'unanimité par le Conseil et à requérir une ratification nationale dans chaque État membre pour pouvoir entrer en vigueur. Le projet prévoyait toutefois plus de flexibilité pour ce qui était de la loi fixant les modalités des ressources de l'Union. Cette dernière pouvait en effet être adoptée par le Conseil à la majorité qualifiée, après approbation du Parlement européen. Ce passage au vote à la majorité qualifiée et l'abolition des ratifications nationales auraient facilité la réforme du rabais budgétaire octroyée au Royaume-Uni ${ }^{90}$.

La CIG a fait marche arrière sur cette question. Désormais, la seconde des lois européennes mentionnées ci-dessus, adoptée par le Conseil à la majorité qualifiée, ne pourra plus que fixer les mesures d'exécution du système de ressources propres de l'Union dans la mesure où la loi fixant les dispositions relatives aux ressources propres le prévoit. Cette loi, elle, sera toujours adoptée à l'unanimité et exigera toujours une ratification nationale ${ }^{91}$. La possibilité d'une procédure de révision plus légère du système des ressources propres est ainsi virtuellement exclue.

\subsection{LE CADRE FINANCIER PLURIANNUEL}

La Convention avait codifié la pratique du cadre financier pluriannuel dans le texte de la Constitution. Le Conseil adoptait ce cadre pluriannuel par un vote à la majorité qualifiée, après approbation du Parlement européen, qui se prononçait à la majorité des membres qui le composent. Ce passage à la majorité qualifiée aurait pu rendre ess discussions budgétaires (quelque peu) moins acrimonieuses. Le projet avait toutefois maintenu l'unanimité pour l'adoption du premier cadre pluriannuel par le Conseil après l'entrée en vigueur de la Constitution.

La CIG a de nouveau opté pour le statu quo. Le cadre pluriannuel continuera ainsi d'être adopté à l'unanimité. Il est néanmoins prévu que le Conseil européen, à l'unanimité, pourra adopter une décision européenne permettant au Conseil d'agir à la majorité qualifiée $^{92}$. L'exigence d'unanimité rend toutefois ce passage à la majorité qualifiée fortement improbable. De plus, les Pays-Bas ont fait une déclaration selon laquelle ils n'accorderont leur consentement à une telle décision de passage à la majorité qualifiée que si une révision de la loi européenne sur les ressources propres fournit préalablement

90 Pour une discussion des options de réforme, cf. W. COUSSENS, «A Fair solution to the UK rebate conundrum », Ideas Factory Europe, Idea 3, téléchargeable de < www.theepc.net>.

91 Article I-54.

92 Article I-55. 
une solution satisfaisante à leur position présumée de contributeur net excessif au budget de l'Union ${ }^{93}$.

En conséquence, les négociations budgétaires continueront à n'être que des querelles acrimonieuses durant lesquelles les États membres n'arrivent à se mettre d'accord que sur le plus petit commun dénominateur.

\subsection{LE BUDGET ANNUEL}

Selon le projet de Constitution établi par la Convention, la distinction entre les dépenses obligatoires et les dépenses non obligatoires devait être supprimée, permettant ainsi au Parlement européen d'avoir le dernier mot en ce qui concerne le budget annuel. La CIG a toutefois remplacé ce mécanisme par une procédure complexe ressemblant à la procédure de co-décision, dans laquelle le Parlement européen conserve néanmoins le dernier mot, comme dans le projet de la Convention ${ }^{94}$.

Selon celle-ci, le budget est initié par la Commission qui présente au Parlement européen et au Conseil un projet regroupant les états provisionnels des dépenses établis par chaque institution. Une fois sa position adoptée, le Conseil la transmet au Parlement. Si ce dernier l'approuve ou ne statue pas, la loi européenne établissant le budget est (réputée) adoptée. Si par contre il adopte des amendements, le projet amendé est transmis au Conseil et à la Commission. Le comité de conciliation ${ }^{95}$ est alors convoqué, sauf si, dans les dix jours de cette transmission, le Conseil informe le Parlement qu'il approuve ces amendements.

Ce comité a pour mission d'aboutir à un accord sur un projet commun à la majorité qualifiée des membres du Conseil et à la majorité des membres représentant le Parlement. S'il n'y parvient pas, un nouveau projet de budget est présenté par la Commission. Si par contre il y parvient, le Parlement et le Conseil disposent chacun d'un certain délai pour l'approuver. Trois voies s'ouvrent alors. Soit, dans ce délai, ces deux institutions approuvent le projet ou ne parviennent pas à statuer ou l'une d'elle l'approuve mais l'autre ne statue pas. Dans ce cas, la loi européenne établissant le budget est réputée adoptée conformément au projet commun. Soit, le Conseil le rejette mais le Parlement l'approuve. Dans ce cas, le Parlement a alors la possibilité de confirmer, à la majorité des membres qui le composent et des trois cinquièmes des suffrages exprimés, les amendements qu'il avait faits antérieurement à la convocation du comité de conciliation ${ }^{96}$. S'il y arrive, la loi européenne établissant le budget est adoptée sur cette base. Soit, enfin, le Parlement et le Conseil rejettent tous deux le projet ou l'une de ces institutions le rejette tandis que l'autre ne parvient pas à statuer ou le Parlement le rejette

93 CIG 87/04 ADD 2, Déclaration du Royaume des Pays-Bas ad article I-55.

94 Article III-404.

95 Le comité de conciliation réunit les membres du Conseil ou leurs représentants et autant de membres représentant le Parlement européen. La Commission participe aux travaux de ce comité et prend toutes les initiatives nécessaires en vue de promouvoir un rapprochement des positions du Parlement et du Conseil (article III-404, \5).

96 Si l'un des amendements du Parlement européen n'est pas confirmé, la position agréée au sein du comité de conciliation concernant la ligne budgétaire qui fait l'objet de cet amendement est retenue (article III-404, S 7, d). 
tandis que le Conseil l'approuve. Dans ces cas, un nouveau projet de budget est présenté par la Commission.

Il faut rappeler par ailleurs que les dispositions du projet de la Convention concernant l'adoption du budget annuel demeuraient ambiguës quant à la question de savoir si le Conseil était censé agir à la majorité qualifiée ordinaire ou à la majorité qualifiée « renforcée » dans cette matière. La CIG a clarifié ce point. Le Conseil agira à la majorité qualifiée normale. 


\section{CONCLUSION}

\section{UNE RÉGRESSION LÉGÈRE MAIS CERTAINE}

La CIG a fait plusieurs pas en arrière par rapport au projet établi par la Convention. Ainsi, bien que d'un point de vue quantitatif, le texte de la Convention ait en grande partie été maintenu, il a été considérablement modifié d'un point de vue qualitatif. En outre, le texte de la CIG est généralement quelque peu plus complexe et obscur que celui de la Convention.

Il y a néanmoins des secteurs où, d'une façon parfois inattendue, la CIG a réussi à apporter une plus-value au texte de la Convention. À titre d'exemples, on pourrait citer la nouvelle version de l'article I-41 (la solidarité contre l'agression armée), l'article I-9 (l'adhésion à la CEDH), l'article III-197 \4 (les décisions du Conseil Ecofin prises uniquement par les membres de la zone euro). Mais ces secteurs sont, comme on peut le constater, moins nombreux.

Les régressions sont, quant à elles, beaucoup plus abondantes. Elles englobent par exemple l'article I-25 (la définition du vote à la majorité qualifiée), l'abrogation de l'article III-63 et la modification de l'article III-62 du projet de Constitution (la dispa rition de la clause passerelle dans le domaine fiscal), les articles III-270 et III-271 (le « frein » dans le domaine de la coopération judiciaire pénale) et l'article III-136 (le « frein » en matière de mesures de sécurité sociale pour les travailleurs migrants).

Pourtant, ceci ne doit pas cacher les faits essentiels. Le résultat final est meilleur que celui qui avait été obtenu à Nice. La CIG a dans quelques cas supprimé certaines ambiguités résultant du texte de la Convention. De plus, le résultat final, bien que clairement insuffisant, est meilleur que ce qu'une CIG «traditionnelle » aurait pu réaliser. Bien qu’il y ait des régressions dans quelques aspects, la CIG ne modifie pas fondamentalement les résultats de la Convention. Le texte de la Convention glissait vers l'intergouvernemental dans ses aspects institutionnels et vers le supranational dans ses politiques. La CIG suit essentiellement la même voie. Le glissement intergouvernemental dans le domaine institutionnel a toutefois été renforcé, tandis que le glissement supranational dans les politiques a, au contraire, été réduit.

\section{UN SYSTÈME INSTITUTIO NNEL LÉGÈREMENT PLUS COMPLEXE ET OBSCUR}

Les changements réalisés par la CIG en matière de dispositions institutionnelles par rapport aux propositions de la Convention renforcent assurément l'aspect intergouvernemental de l'équilibre institutionnel. Les seuils de majorité qualifiée ont, premièrement, été relevés. Cela renforce dès lors le pouvoir des États membres et, en particulier, des États membres les plus peuplés. La portée du vote à la majorité qualifiée 
a, deuxièmement, été réduite, comme l'a aussi été la possibilité de recours aux « valves de sécurité », c'est-à-dire aux clauses passerelle.

Troisièmement, la capacité de la Commission de déterminer les priorités de l'agenda pourra être affectée par les programmes pluriannuels des présidences jointes. Quatrièmement, la Commission sera formée selon une logique intergouvernementale jusqu'à 2014 et la réduction par la suite de sa taille pourra être bloquée au vu de l'exigence de rotation égale entre les États membres. Cinquièmement, les pouvoirs du président de la Commission pour choisir son équipe sont réduits, puisqu'il ne pourra plus choisir au sein d'une liste de trois candidats. Sixièmement, les modifications apportées aux dispositions relatives au ministre des Affaires étrangères laissent présager qu'il écoutera prioritairement les demandes du Conseil. Finalement, le progrès limité de la Convention concernant la décision sur les ressources propres et le cadre financier pluriannuel a été nuancé par la CIG.

Les modifications apportées par la CIG ne servent pas non plus les causes de la démocratie et de la transparence. Le rôle du Parlement européen a été réduit dans quelques cas (par exemple pour les décisions budgétaires). Le système de vote à la majorité qualifiée a été rendu plus opaque. Le Conseil législatif - un outil permettant un contrôle accru de la responsabilité du Conseil - a été supprimé.

Finalement, bien que la lutte entre les grands et les petits États membres semble avoir été résolue par l'adoption du traité, cela n'est, en partie, qu'une apparence. En effet, le règlement de beaucoup de sujets de discussion importants à cet égard (par exemple la composition du Parlement européen) a souvent été simplement reporté. De plus, la distribution de la puissance de vote résultant de la nouvelle définition de la majorité qualifiée et en particulier des éléments en faveur des plus grands États membres, pourrait inciter des appels à une nouvelle révision du texte à l'avenir. Ce qui pourrait être également le cas de la composition de la Commission.

\section{DES POLITIQUES LÉGÈREMENT MOINS EFFICACES}

Dans le domaine de l'UEM, les prérogatives de la Commission ont été réduites par rapport au projet de la Convention. Le rôle des États de la zone euro a toutefois été renforcé, mais comme nous l'avons expliqué, cela ne peut être vu comme une panacée. Il aurait en effet été plus important de préserver (voire de renforcer) le rôle de la Commission.

Les progrès substantiels de la Convention dans le domaine de la Justice et des Affaires intérieures ont été partiellement réduits. Des « freins » ont été prévus dans le secteur de la coopération judiciaire pénale. Les prérogatives d'Eurojust et du procureur européen ont été réduites.

En ce qui concerne les relations extérieures de l'Union, la CIG a clarifié et a facilité le fonctionnement de la coopération structurée (permanente). Toutefois, le Conseil ne peut plus demander aux États membres participant à cette coopération d'effectuer une mission de la Politique de sécurité et de défense commune. L'autorisation pour une coopération renforcée dans le secteur PESC exige maintenant l'unanimité du Conseil et 
la portée du vote à la majorité qualifiée dans la politique commerciale commune a été réduite.

Quant à la politique sociale, la CIG a prévu une procédure de freinage en matière de sécurité sociale des travailleurs migrants. La nature de la compétence de l'Union apparait finalement très confuse. De plus, les références à la clause de cohérence et au sommet social tripartite peuvent s'avérer être une simple façade cachant une Union loin des préoccupations sociales. Finalement, le petit progrès réalisé par la Convention dans le domaine de la fiscalité a été complètement éliminé.

\section{UNE PROCÉDURE DE RÉVISION DONT LA RÉFORME S'IMPOSE ${ }^{97}$}

\section{La création de la Convention a été positive, mais a eu un prix en termes de complexité et n'est clairement pas suffisante}

Il est important de tirer des leçons du processus de révision de 2002-2004. En effet, le Traité constitutionnel prévoit maintenant que les futures révisions du traité pourront aussi être préparées par une Convention. La création de la Convention par la déclaration de Laeken a clairement été une avancée, mais des problèmes importants subsistent. Premièrement, les débats de la Convention sont actuellement (et le restent dans le Traité constitutionnel) une étape supplémentaire du processus de révision traditionnel et non pas une modification de ce processus. Deuxièmement, même si la Conv ention constitue en elle-même une amélioration, elle augmente la longueur et la complexité du processus de révision. Troisièmement, même à l'heure actuelle, le recours à une Convention ne diminue pas fortement les pressions traditionnelles de toute procédure de révision. En synthèse, c'est un premier pas mais l'Union européenne a besoin de quelque chose de mieux, à savoir des mécanismes qui abandonnent l'unanimité au stade tant de l'élaboration que de l'approbation des traités.

La CIG a mis en évidence la force des intérêts nationaux, même à très courte vue. Avec les procédures décisionnelles actuelles, c'était probablement inévitable, d'autant plus que l'ombre de la nécessité de ratifications nationales unanimes colore toujours toute négociation. Cependant, cela rend très probable le fait que les révisions futures du Traité constitutionnel ressembleront assez fort aux processions d'Echternach, avec la Convention avançant de deux pas et la CIG reculant d'un pas. Cet effet aura dès lors des conséquences évidentes sur la stratégie à suivre durant les Conventions.

97 Pour une analyse de ce problème et du résultat de la Convention dans ce domaine, cf. F. DEHOUSSE et W. COUSSENS, "Rethinking the revision and entry into force clauses of the European Constitution », F. DEHOUSSE et W. COUSSENS (réd.), The Convention's Constitutional Treaty : Old wine in a new bottle?, op. cit. 


\section{L'impact réel de l'élargissement sur le processus de révision n'a toujours pas été mesuré}

On l'a peu remarqué dans le chaos général, mais l'élargissement a des conséquences substantielles sur le processus de révision, ne serait-ce que parce qu'il rend plus difficile un accord entre tous les États membres. Cette première difficulté pourrait d'ailleurs être amplifiée lors de la phase des ratifications nationales du Traité constitutionnel ${ }^{98}$.

Pour que le Traité constitutionnel entre en vigueur, il doit en effet toujours être ratifié par chaque État membre, que ce soit par voie parlementaire ou par référendum. Au moins huit États membres vont probablement organiser un référendum. Le risque qu'au moins un État membre refuse le texte du nouveau traité est dès lors considérable. Si cela ne concerne que des petits pays et que le nombre de pays votant non reste limité, il semble probable que l'on demandera alors à ces pays d'essayer une deuxième fois, probablement après quelques modifications au traité, c'est-à-dire un scénario identique à celui qui fut suivi en 1992 avec le Danemark et en 2001 avec l'Irlande. Dans le cas contraire, l'entrée en vigueur du traité pourrait toutefois bien être bloquée pour longtemps.

La Convention avait pris ce problème en considération en prévoyant la réunion du Conseil européen en cas de problèmes lors des ratifications nationales. En effet, si deux ans après la signature du Traité constitutionnel, quatre cinquièmes des États membres l'ont ratifié et un ou plusieurs États membres ont rencontré des difficultés dans le processus de ratification, la question doit être, selon la Constitution, traitée par le Conseil européen ${ }^{99}$. Cela ne constitue évidemment pas une solution réelle à ce problème.

On avait proposé plusieurs autres solutions pendant la Convention. Aucune n'a toutefois été retenue, entre autres raisons parce que leur valeur légale était plutôt faible. En effet, le nouveau traité étant la suite des traités actuels, il doit respecter leurs procédures de révision, qui prévoient un accord unanime et une ratification nationale. C'est donc à la précédente CIG que l'on peut reprocher d'avoir manqué l'occasion de supprimer cette exigence excessive de révision unanime du traité.

Et pourtant, on n'a pas tiré de leçon d'une telle erreur. Tant la Convention que la CIG ont en effet échoué à créer une procédure plus flexible pour les futures révisions, bien que, cette fois-ci, cela ait été parfaitement possible d'un point de vue légal. La CIG s'est ainsi bornée à établir que pour les futures révisions du traité aucune Conférence intergouvernementale ne devra être convoquée s'il s'agit d'une révision des dispositions de la partie III sur les politiques internes qui n'augmente pas les compétences conférées à l'Union. Dans ce cas, le Conseil européen aura en effet le droit de décider de telles révisions par un vote unanime. Ces révisions n'entreront toutefois en vigueur qu'après leur ratification unanime par les États membres ${ }^{100}$. C'est évidemment un changement assez mineur comparé au statu quo.

98 Pour une analyse plus détaillée, cf. F. DEHOUSSE et W. COUSSENS, "The Enlargement of the European Union - Opportunities and Threats », Studia Diplomatica, 2001, n ${ }^{\circ} 4$, pp. 1-139.

99 CIG 87/04 ADD 2, Déclaration concernant la ratification du Traité établissant une Constitution pour l'Europe.

100 Article IV-445. 
Tout cela illustre l'impasse de l'approche actuelle des États membres: l'unanimité et le droit international traditionnel sont devenus complètement inadaptés aux réalités d'une Europe intégrée comptant 25 États membres et 500 millions d'habitants. Il est à cet égard tout à fait compréhensible que la CIG ait décidé d'enlever de la Constitution la phrase de Thucydide, affirmant: «Notre Constitution (...) est appelée démocratie parce que le pouvoir est entre les mains non d'une minorité, mais du plus grand nombre.» L'exigence de révision du traité à l'unanimité mène en réalité exactement au résultat opposé. Cette phrase constituait la meilleure incrimination imaginable du projet réalisé par la Convention.

Bien qu'un pas dans la bonne direction, le projet de Constitution de la Convention, était déjà inadéquat pour adapter les institutions européennes aux nouvelles réalités de l'élargissement, la CIG a légèrement aggravé cette inadéquation. Dans de telles circonstances, il devient nécessaire soit d'accepter une réforme plus profonde du processus de révision, soit d'insister sur une utilisation accrue des mécanismes de flexibilité ${ }^{101}$.

101 Cf. F. DeHOusse et W. COUSSENS (réd.), The hopes and limits of flexible integration in the future Europe, Studia Diplomatica, op. cit., pp. 1-77. 


\section{Derniers Courriers hebdomadaires parus}

1818-1819- Le groupe de travail politique (24 octobre 1962 - 24 octobre 1963). Jalons d'une

1820 évolution institutionnelle Par Alain Stenmans

1821-1822 Le secteur des télécommunications en Belgique : quel bilan après cinq ans de concurrence?

Par Franklin Dehousse, Tania Zgajewski

1823-1824 La carrière des chercheurs dans les universités de la Communauté française Par Pierre Feyereisen, Sophie Huyghues-Despointes, Maria Oliver Burguera

1825 Index 2003

1826-1827 La Confédération européenne des syndicats. Résolutions du congrès de Prague et position du syndicalisme belge Par Corinne Gobin

$1828 \quad$ La responsabilité sociale des entreprises Par Anne Peeters

1829-1830 Les programmes électoraux des partis transnationaux pour l'élection du Parlement européen de juin 2004

Par Thierry Coosemans

1831 Les positions des partis politiques francophones en matière d'enseignement pour les élections du 13 juin 2004

Par Julien Nicaise

1832 Les programmes des partis francophones pour l'élection du Parlement européen de juin 2004

Par Thierry Coosemans

1833-1834 La préparation des élections du 13 juin 2004. I. Réformes électorales et rappel des élections antérieures

Par Étienne Arcq, Pierre Blaise, Vincent de Coorebyter

1835-1836 La préparation des élections du 13 juin 2004. II. Les listes en présence

Par Étienne Arcq, Pierre Blaise, Caroline Sägesser

1837-1838 Débats parlementaires bruxellois (1999-2004). I. Les relations communautaires, la fiscalité et la mobilité

Par Jean-Paul Nassaux

1839-1840 Débats parlementaires bruxellois (1999-2004). II. Les nuisances sonores, la politique de l'eau et la Cobru Par Jean-Paul Nassaux

1841-1842 La création du Service fédéral des créances alimentaires. Histoire d'une revendication

Par Marie-Thérèse Coenen

1843-1844 L'élection du Parlement européen de juin 2004

Par Thierry Coosemans

1845-1846 L'évolution récente des relations transatlantiques Par Inès Trépant 